\title{
Optical Phase Conjugation in Installed Optical Networks
}

\author{
G. Saavedra $^{(1)}$, Y. Sun ${ }^{(2)}$, K. R. H. Bottrill ${ }^{(2)}$, L. Galdino ${ }^{(1)}$, F. Parmigiani( ${ }^{(2)}$, Z. Liu ${ }^{(1)}$, D.J. Richardson ${ }^{(2)}$, \\ P. Petropoulos ${ }^{(2)}$, R. I. Killey ${ }^{(1)}$, and P. Bayvel ${ }^{(1)}$ \\ (I) Optical Networks Group, Dept. of Electronic \& Electrical Engineering, UCL, Torrington Place, London, WC1E 7JE, UK \\ (2) Optoelectronics Research Centre, University of Southampton, Southampton, SO17 1BJ,UK \\ uceegs@ucl.ac.uk,.ys9g11@ecs.soton.ac.uk
}

\begin{abstract}
We demonstrate a record throughput of $5.7 \mathrm{Tbit} / \mathrm{s}$ employing an optical phase conjugator to jointly compensate chromatic dispersion and increase the nonlinear threshold in an installed optical network using commercially available lumped amplifiers.

OCIS codes: (060.4510) Optical communications; (060.4370) Nonlinear optics, fibers.
\end{abstract}

\section{Introduction}

Management of the dispersion and optical nonlinearity remain important challenges in high-capacity transmission systems. Even though electronic dispersion compensation (EDC) has proven to be robust for the compensation of dispersive effects, mitigation of nonlinearity-induced impairments in WDM environment is still problematic. Digital nonlinear compensation techniques are currently limited by the bandwidths available for coherent receivers. Optical phase conjugation (OPC) has shown considerable promise for the simultaneous compensation of both dispersion and nonlinearity when placed at the mid-point of a fiber-optic transmission link. It has been shown not only to improve the signal quality significantly in purpose-built, symmetrised laboratory settings [1], but to also benefit erbium-doped fiber amplifier (EDFA)-based field-installed transmission systems that do not adhere to the strict design rules of OPCbased transmission [2].

An important benefit of four-wave mixing based OPC is that it is transparent to the modulation format and lends itself to multi-channel operation. As with many all-optical processing systems, OPC-based transmission becomes more economically attractive as the number of WDM channels that are processed in the phase conjugator increases. To date, WDM experiments of up to six polarization multiplexed (PM) channels carrying 64-QAM signals have been demonstrated [3], one recent experiment with a single polarization multiplexed (PM) 256-QAM channel. Both demonstrations used dispersion-managed transmission links [4].

In this work, we investigate the OPC performance in a 440-km EDFA-based non-dispersion-compensated fieldinstalled transmission experiment up to 14 PM Nyquist-WDM channels, each carrying 40 GBd 256-QAM signal. The results show that the operable window of launch power per channel is increased by $1 \mathrm{~dB}$ when the OPC is in place as compared to the case of electronic dispersion compensation (EDC) at the receiver, indicating effective mitigation of nonlinearity for all the evaluated bandwidths.

\section{Transmission setup}

The transmission setup used in the experiment is shown in Fig. 1The transmitter, located in London, was formed by 14 external cavity lasers (ECLs). The wavelengths used were divided in two bands to optimize OPC operation, with

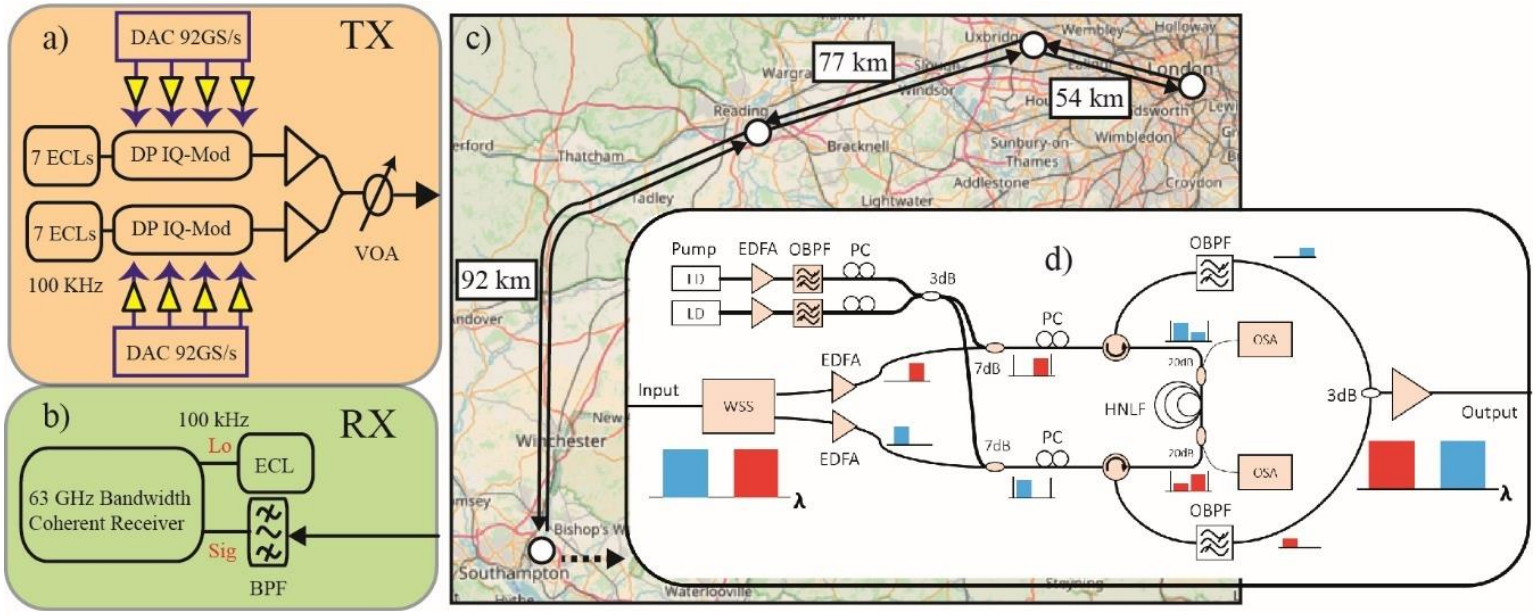

Fig. 1, Experimental setup. a) Transmitter, b) Receiver, c) Transmission link, d) Optical phase conjugator (Map inset @ Open Street Map) 
a short wavelength band between 1552.12 and $1554.13 \mathrm{~nm}$, and a long wavelength band between 1558.29 and $1560.20 \mathrm{~nm}$. In both bands, the spacing between the lasers was $41 \mathrm{GHz}$. Subsequently, the ECLs were modulated by dual polarization IQ modulators, driven by the electrically amplified output from $92 \mathrm{GS} / \mathrm{s}$ digital-to-analogue converters (DACs). The drive signals were generated off-line and corresponded to $40 \mathrm{GBd}$ 256-QAM shaped using a root raised cosine filter with a $1 \%$ roll-off factor. After modulation, the signals were optically amplified and coupled together using a $3 \mathrm{~dB}$ coupler to form two 7xPM-256QAM super-channels.

Transmission was carried out on the National Dark Fibre Infrastructure Service (NDFIS) connecting UCL and the University of Southampton via two intermediate sites [5]. Optical amplification was carried out by EDFAs and no dispersion compensating modules were used. Optical phase conjugation was performed at the University of Southampton, corresponding to the middle of the link.

The main features of the OPC module include insensitivity to the polarization of the incoming signal(s) and efficient bandwidth utilization in a single nonlinear medium $[2,3]$. Polarization insensitivity was enabled by an orthogonally polarized dual-pump four-wave mixing (FWM) configuration. Bandwidth efficiency was ensured by splitting the transmitted channels into two bands, each band defined by the position of one pump and the average frequency of the two pumps, and swapping their spectral positions through propagation in opposite directions of the highly-nonlinear fiber (HNLF). At the output, the original signals were filtered out, and the phase-conjugated idlers occupied the wavelengths of the opposite band. The two continuous-wave pumps used in our experiments were separated by $12 \mathrm{~nm}(1.5 \mathrm{THz})$, and were located at 1550.12 and $1562.23 \mathrm{~nm}$. They were amplified separately and filtered to suppress the out-of-band amplified spontaneous emission noise. A pair of polarization controllers ensured orthogonality of their states of polarization. After the two pump waves were combined in the same fiber, they were split into two copies, directed to the two opposite ends of the HNLF, combined this way with each one of the signal bands (see Fig.1). The power per pump per direction was $22 \mathrm{dBm}$. The HNLF was a $101 \mathrm{~m}$ strained germanium-doped silica fiber with a zero-dispersion wavelength of $1531 \mathrm{~nm}$, a dispersion slope of $0.018 \mathrm{ps} / \mathrm{nm}^{2} / \mathrm{km}$, a polarization mode dispersion of $0.31 \mathrm{ps} / \mathrm{km}^{1 / 2}$, and a nonlinear coefficient of $11.6 / \mathrm{W} / \mathrm{km}$. The conversion efficiency, defined as the idler output to signal input power ratio, of the FWM process was approximately $-10 \mathrm{~dB}$.

At the output of the transmission link, the signals were amplified and a band-pass filter was used to select the channel to be detected. The receiver was comprised of an independent ECL used as local oscillator, and detection was carried out in a digital coherent receiver followed by digital signal processing (DSP). When no OPC was used in the middle of the link, electronic dispersion compensation (EDC) was carried out after detection, otherwise this step was not performed. The DSP chain used after is detailed in [6]. Finally, signal-to-noise ratio (SNR) and mutual information were calculated using the transmitted and received symbols after DSP.

\section{Results and discussion}

The transmission performance of the system is seen Fig. 2, where the SNR is plotted as a function of the launch power per channel for two different transmitted bandwidths, corresponding to the transmission of 3 (between 1553.1 and $1554.13 \mathrm{~nm}$ ) and 14 channels. In both cases, in the linear regime when OPC is used a small penalty is introduced due the negative conversion efficiency of the setup. Additionally, OPC increases the nonlinear tolerance of the transmission by compensating nonlinear distortions generated by the Kerr nonlinearity. For both transmitted
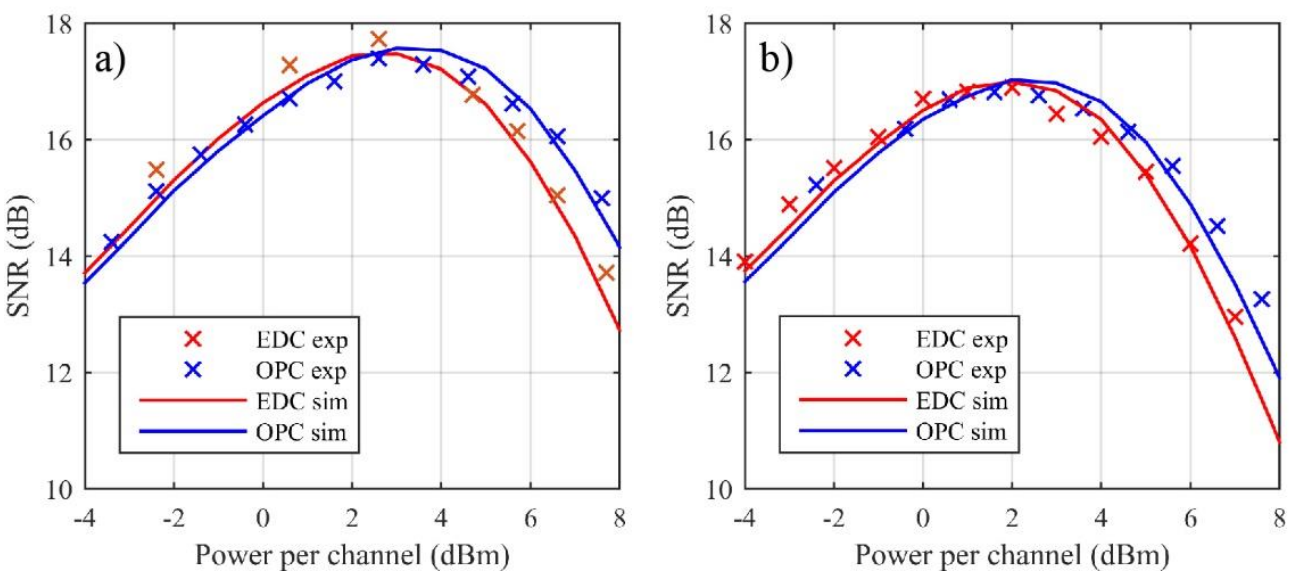

Fig. 2, Signal-to-noise ratio as a function of signal launch power for the transmission of a) 3 channels, b) 14 channel. Markers represent experimental results and solid lines numerical simulations. 
bandwidths, the optimum launch power is increased by approximately $1 \mathrm{~dB}$, however, the maximum SNR compared to EDC did not improve. The observed gain is limited by the properties of the transmission link, the use of EDFA and power asymmetry relative to the conjugation point. Numerical simulations were performed to corroborate the performance with excellent agreement in both cases. Optical networks often employ different span lengths to connect different nodes, further reducing the power symmetry, and thus the gain from the compensation of nonlinear effects.

Our experiment suggests that the OPC configuration we used allows processing of an increased number of transmitted channels without introducing signal degradation by the generation of parasitic higher order idlers during the conjugation process. Additional benefits offered by OPC relate to the simultaneous compensation of chromatic dispersion for all the transmitted WDM channels within the transmission link, and as the number of channels is increased this benefit can be exploited to reduce the power consumption of the receivers and reduce transmission latency.

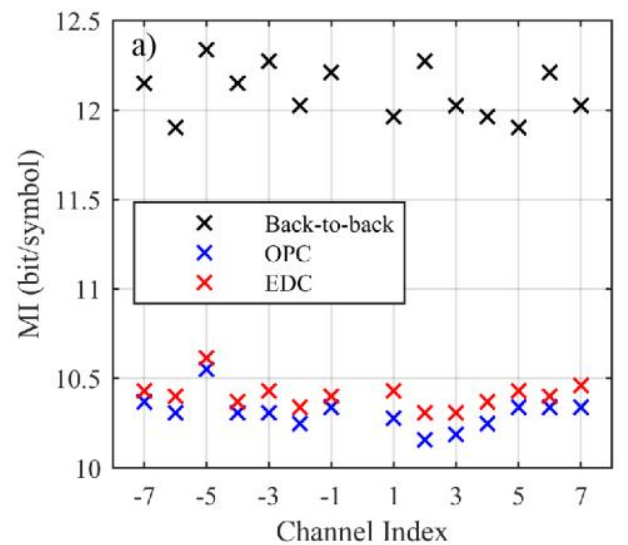

b)

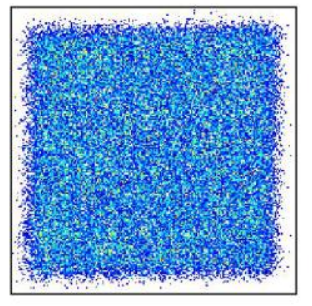

Back-to-back c)

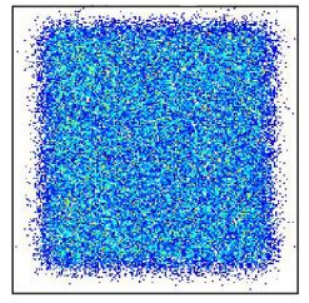

EDC d)

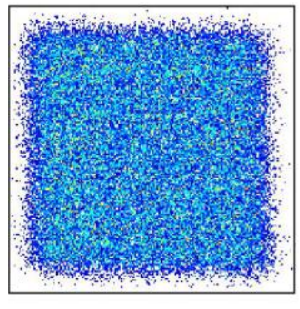

OPC

Fig. 3, a) Mutual information for all transmitted channels. Received constellations of the channel -5 for b) back-to-back c) EDC and d) OPC

The performance of the transmission using OPC was evaluated for all the transmitted channels using mutual information as a metric, allowing therefore to analyze the achievable information rates. The use of 14 PM256QAM channels is translated into the large throughput transmitted using OPC and corresponds to $5.7 \mathrm{~Tb} / \mathrm{s}$. Similar performance was observed in all the transmitted channels, confirming that no additional penalties were observed from the OPC when the bandwidth was increased.

\section{Conclusion}

The use of optical phase conjugation in dispersion-uncompensated lumped-amplified installed optical networks was numerically and experimentally analyzed using $14 \mathrm{x}$ WDM 40Gbaud channels modulated with PM-256 QAM, corresponding to the largest bandwidth and throughput conjugated in transmission. The experiment was performed over an installed fiber transmission link, where phase conjugation was performed at a remote site from the transmitter and receiver. The use of different span lengths in the transmission was identified as one factor limiting the gain offered by OPC from nonlinear compensation. No performance reduction was observed as the signal bandwidth was increased from $120 \mathrm{GHz}$ to $560 \mathrm{GHz}$, confirming the suitability of OPC for WDM operation.

This experiment has been performed on the jointly EPSRC/JISC supported NDFIS. This work has been supported by Becas Chile, and the UK EPSRC UNLOC and Photonics Hyperhighway Programme Grants. The authors would like to thank Oclaro for the High Bandwidth Polarization Multiplexed Quad Mach-Zehnder Modulator

\section{References}

[1] K. Solis-Trapala, et al., "Optimized WDM Transmission Impairment Mitigation by Multiple Phase Conjugations," J. Lightwave Technol. 34 , 431-440 (2016)

[2] Y. Sun et al., "Optical Phase Conjugation for Simultaneous Dispersion and Nonlinearity Compensation Performed over an 800-km long Field-installed Transmission Link" in Proc. ECOC, Th.1.F.2, Gothenburg (2017).

[3] S. Yoshima et al., "Mitigation of Nonlinear Effects on WDM QAM Signals Enabled by Optical Phase Conjugation With Efficient Bandwidth Utilization," in Journal of Lightwave Technology, vol. 35, no. 4, pp. 971-978, 2017

[4] F. Da Ros. et al., "Impact of Signal-Conjugate Wavelength Shift on Optical Phase Conjugation-based Transmission of QAM Signals," in Proc. ECOC, P1.SC4.66, Gothenburg (2017).

[5] The National Dark Fiber Infrastructure Service (NDFIS) [Online]. Available: http://www.ndfis.org/

[6] G. Saavedra et al., "Experimental Analysis of Nonlinear Impairments in Fibre Optic Transmission Systems up to 7.3 THz", to appear in Journal of Lightwave Technology, DOI: 10.1109/JLT.2017.2760138 